\title{
Exploring polarization features in light reflection from beetles with structural colors
}

\author{
Hans Arwin $^{a}$, Roger Magnusson ${ }^{a}$, Lía Fernández del Río ${ }^{a}, \operatorname{Jan}_{\text {Landin }}{ }^{a}$, \\ Arturo Mendoza-Galván ${ }^{a, b}$ and Kenneth Järrendahl ${ }^{a}$ \\ ${ }^{a}$ Department of Physics, Chemistry and Biology, Linköping University, Linköping, Sweden; \\ ${ }^{b}$ Cinvestav-IPN, Unidad Querétaro, Libramiento Norponiente 2000, 76230 Querétaro, Mexico
}

\begin{abstract}
A Mueller matrix of a sample can be used to determine the polarization of reflected light for incident light with arbitrary polarization. The polarization can be quantified in terms of ellipticity, polarization azimuth and degree of polarization. We apply spectroscopic Mueller-matrix ellipsometry at multiple angles of incidence to study the cuticle of beetles and derive polarization features for incident unpolarized light. In particular we address chiral phenomena in scarab beetles, the origin of their structural colors and the observed high degree of circular polarization is discussed. Results from beetles in the Scarabaeidae subfamilies Cetoniinae and Rutelinae are presented including specimens with broad-band silver- or gold-like colors with metallic shine as well as specimens with narrow-band green or red reflectors. The variation of polarization with angle of incidence and occurrence of both left-handed and right-handed polarization from a single species are presented. We also use Mueller-matrix spectra in electromagnetic modeling and show how to determine structural parameters including cuticle layer thicknesses and optical properties. Interference oscillations in the observed spectra are due to allowed optical modes and we show how to develop a structural model of a cuticle based on this effect. Sum decomposition of Mueller matrices measured on a depolarizing cuticle of a beetle is briefly discussed.
\end{abstract}

Keywords: Mueller-matrix ellipsometry, chiral reflectors, circular polarization, beetle cuticle

\section{INTRODUCTION}

Natural light reflected from the cuticle of several species of beetles may exhibit beautiful colors and some beetles have metallic shine. As examples we show in Fig. 1 some of the beetles studied in this investigation. The origin of these colors may be absorption of light by cuticle pigments in parts of the visible/ultraviolet spectrum but in many cases the colors are due to the structure of the outer parts of the cuticle. We will here focus on these so-called structural colors. Mechanisms for the structural color generation in the cuticle of beetles have been reviewed by Seago et al. ${ }^{1}$ and include multilayer reflectors, three-dimensional photonic crystals, diffraction gratings and scattering. However, in addition to color and irradiance, the reflected light has an additional and important attribute which is polarization. The human eye is essentially blind to polarization but many animals, primarily among arthropods, chordates and molluscs, have vision with polarization sensitivity. ${ }^{2}$ A fascinating example is the beetle Scarabaeus zambesianus which can use the polarization of a moonlit sky for navigation. ${ }^{3}$

The polarization sensitivity of animal vision is often indirect in the sense that the response of their photoreceptors depends on polarization but there is no recognition of polarization as a specific property of light. The latter, i.e. a detection of polarization property, would correspond to a polarimetric measurement and can be found in some animals with single polarization-sensitive photo-receptors. By movements of the eyes or the head, successive responses can be used to discriminate among different polarization states. However, a detailed understanding of the role of polarization in animal vision is not developed yet. So far most research has been focused on linear polarization. ${ }^{2}$

When it comes to polarization by reflection, which is addressed here, we have to consider that reflection of light on most surfaces changes the state and/or the degree of polarization. For bioreflectors, the polarization

Further author information: (Send correspondence to H. Arwin)

H. Arwin: E-mail: han@ifm.liu.se, Telephone: +46 13281215

Bioinspiration, Biomimetics, and Bioreplication 2015, edited by Akhlesh Lakhtakia,

Mato Knez, Raúl J. Martín-Palma, Proc. of SPIE Vol. 9429, 942909 · — 2015 SPIE

CCC code: $0277-786 \mathrm{X} / 15 / \$ 18 \cdot$ doi: $10.1117 / 12.2083032$

Proc. of SPIE Vol. 9429 942909-1 

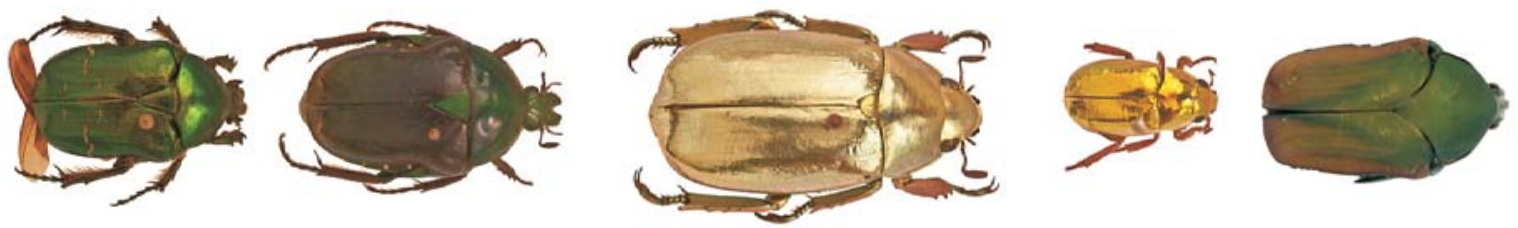

Figure 1. Photos of the beetle species studied in this report. From left to right: Cetonia aurata, Coptomia laevis, Chrysina argenteola, Anoplognathus aureus, Cotonis mutabilis. The length of C. argenteola is $29 \mathrm{~mm}$ and the other beetles are shown on the same scale. (Photos: Jens Birch and author A. M-G. (C. mutabilis)).

may therefore be a side effect and the biological relevance of polarization is therefore not obvious and is under debate. Some possible functions of polarization may be intraspecies communication, to avoid predators by hiding or to scare by mimicking a poisonous material. Brady and Cummings ${ }^{4}$ investigated effects of polarization and found that the scarab beetle Chrysina gloriosa (Leconte, 1854) can detect and respond differently to circularly polarized light. However, Blahó et al. ${ }^{5}$ studied several species of Scarab beetles to test if they are attracted to circularly polarized light when looking for food or a mate and found that the polarization has no influence. For some butterflies it has been shown that polarized light is important as a mating signal. ${ }^{6}$ However, for more complex polarization features, like near-circular polarization, it is in our opinion likely that such features have evolutionary advantages which need to be more investigated. Near-circular polarization in light reflected from the cuticle of beetles were discovered more than 100 years ago by Michelson ${ }^{7}$ who also claimed that both left-handed and right-handed polarization could be found in light reflected from the cuticle of beetles. These findings were questioned for more than half a century but finally it was proven that Michelson was correct. ${ }^{8}$ More recently these effects have been quantified using advanced optical measurement techniques. ${ }^{9-11}$

By measuring the normal-incidence spectral reflectance of a beetle cuticle, we essentially acquire a quantification of its color. ${ }^{12,13}$ A more complete description requires that the angle of incidence is varied, that the polarization of the incident light is controlled and that the polarization of the reflected light is measured including its degree of polarization. Mueller-matrix spectroscopic ellipsometry (MMSE) can provide such complete characterization and we here use MMSE to explore polarization features in beetle cuticle reflection and to understand their physical origin through electromagnetic modeling.

Our long-term goal is to present a comprehensive base of knowledge about light polarizing capabilities of the cuticle of beetles and contribute to the understanding of the physical origin of these features. Such information is of large fundamental interest for understanding insect behavior, ecology and evolution. ${ }^{14}$ There is also an economical and environmental motivation as such information may facilitate development of alternatives to insecticides for crop protection against beetles. Last but not least there is a technological interest in the area of biomimetics. ${ }^{15-17}$ A beetle cuticle is multifunctional and may combine several physical properties like color, polarization, hardness, low weight, self-cleaning, etc. Considering that their constituents mainly are carbohydrates and proteins which are environmentally friendly, it is very attractive to mimic such bioreflectors and develop applications like decorative coatings, polarization filters, self-cleaning surfaces, sensor devices and more.

In this report our objective is limited to discuss near-circular polarization effects in beetles in the Scarabaeidae subfamilies Cetoniinae and Rutelinae as observed by MMSE. More specifically we present both narrow-band and broad-band near-circularly polarizing bioreflectors in the cuticle of beetles. Variations in polarization with angle of incidence, including handedness will be discussed. We will also demonstrate how MMSE data can be utilized to develop and analyze structural models of a beetle cuticle. The polarization properties we present are only a representative selection as it would be too much to report resulting polarization states in reflected light for all possible incident polarizations for each beetle.

\section{THEORY}

The methodology we use is based on so-called Stokes-Mueller formalism for description of light and its interaction with samples. A brief introduction to Stokes vectors, Mueller matrices and how they can be used to derive polarization parameters is given here. Further details can be found elsewhere. ${ }^{18}$ 


\subsection{Stokes vectors and Mueller matrices}

A Stokes vector $\mathbf{S}$ provides a description of a light beam and includes its irradiance, polarization and degree of polarization. In an $x y z$ Cartesian coordinate system with $z$ as the direction of light propagation, a Stokes vector is defined as ${ }^{18}$

$$
\mathbf{S}=\left[\begin{array}{c}
I \\
Q \\
U \\
V
\end{array}\right]
$$

where $I$ is the total irradiance, $Q$ represents the difference between the irradiances in the $x$ - and $y$-directions, i.e. the preference to either $x$ - or $y$-polarization, $U$ represents the difference between the irradiances in the $+45^{\circ}$ and the $-45^{\circ}$ directions of linear polarization and $V$ represents the difference between the irradiances of the right-circular state and the left-circular state of polarization*.

From the Stokes parameters $I, Q, U$ and $V$ we can derive several parameters describing the polarization of the light. The degree of polarization is given by

$$
P=\frac{\sqrt{Q^{2}+U^{2}+V^{2}}}{I}
$$

This may be refined to the degree of circular polarization

$$
P_{\text {circ }}=\frac{V}{I}
$$

The degree of circular polarization is more correctly defined as $P=\sqrt{V^{2}} / I$ and should have a value in the range $[0,1]$. However, to include handedness in $P$ we use Eq. (3) and extend the range of $P$ to $[-1,1]$. Another useful measure is the ellipticity angle

$$
\varepsilon=\frac{1}{2} \arcsin \frac{V}{\sqrt{Q^{2}+U^{2}+V^{2}}}
$$

For circular polarization we have $\varepsilon= \pm 45^{\circ}$ and also here we include handedness with + for right-handedness and - for left-handedness.

The polarizing properties of a beetle cuticle are investigated by analyzing its Mueller matrix $\mathbf{M}$, which in its normalized form is given by ${ }^{18}$

$$
\mathbf{M}=\left[\begin{array}{cccc}
1 & m_{12} & m_{13} & m_{14} \\
m_{21} & m_{22} & m_{23} & m_{24} \\
m_{31} & m_{32} & m_{33} & m_{34} \\
m_{41} & m_{42} & m_{43} & m_{44}
\end{array}\right]=\left[\begin{array}{cc}
1 & \mathbf{D}^{T} \\
\mathbf{P} & \mathbf{m}
\end{array}\right]
$$

where we also have introduced the polarizance vector $\mathbf{P}=\left[m_{21}, m_{31}, m_{41}\right]^{T}$ ( $T$ indicates transpose), the diattenuator vector $\mathbf{D}=\left[m_{12}, m_{13}, m_{14}\right]^{T}$ and the short notation $\mathbf{m}$ for the $3 \times 3$ matrix. Normalization of the measured Mueller matrix is done by dividing all elements with the value of element $M_{11}$ of the originally measured Mueller matrix.

The matrix $\mathbf{M}$ can be determined in an ellipsometric experiment from the following relation

$$
\mathbf{S}_{o}=\mathbf{M S}_{i}
$$

where $\mathbf{S}_{o}$ and $\mathbf{S}_{i}$ are Stokes vectors of reflected and incident light, respectively. To determine all elements of $\mathbf{M}$ it is necessary to perform measurements of $\mathbf{S}_{o}$ at several $\mathbf{S}_{i}$.

\footnotetext{
${ }^{*}$ We use the convention that light is right-handed polarized for clock-wise rotation of the electric field when looking towards the source.
} 

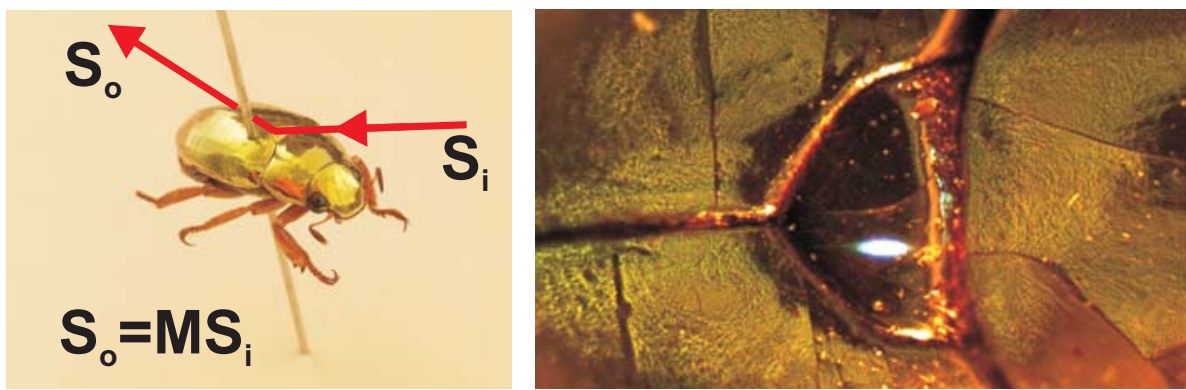

Figure 2. Illustration of a measurement (left). The spot size on the beetle scutellum is seen as a small bright spot (right).

\subsection{Derived parameters}

From a Mueller matrix we can determine several characteristic polarization features of a reflecting surface. By specifying the incident Stokes vector $\mathbf{S}_{i}$ we can model all possible states of the specularly reflected light by analyzing $\mathbf{S}_{o}$ from Eq. (6). We will here only explore cuticle properties in unpolarized light, i.e. only $\mathbf{S}_{i}=I[1,0,0,0]^{T}$ will be used. From Eq. (6) we then find that only the first column in $\mathbf{M}$ is needed and we get $\mathbf{S}_{o}=I\left[1, m_{21}, m_{31}, m_{41}\right]^{T}$. Equations (2)-(4) then reformulate to

$$
\begin{aligned}
P & =\sqrt{m_{21}^{2}+m_{31}^{2}+m_{41}^{2}} \\
P_{\text {circ }} & =m_{41} \\
\varepsilon & =\frac{1}{2} \arcsin \frac{m_{41}}{\sqrt{m_{21}^{2}+m_{31}^{2}+m_{41}^{2}}}
\end{aligned}
$$

\section{EXPERIMENTAL DETAILS}

\subsection{Mueller-Matrix Ellipsometry}

Spectral Mueller-matrix measurements were performed with a dual rotating compensator ellipsometer (RC2) from J. A. Woollam Co., Inc. for wavelengths $\lambda$ in the spectral range 300-1000 nm at multiple angles of incidence $\theta$ in the range $20^{\circ}-75^{\circ}$. With long-focus optics a spot size of 50-100 $\mu \mathrm{m}$ depending on $\theta$ was achieved. Each beetle is pinned and the pin was used to mount the beetle on the sample holder having a rotation stage and an $x y$-translator. With a camera the actual measurement position on the cuticle could be observed from scattered light as shown in Figure 2. The accuracy of the measured Mueller-matrix elements $m_{i j}$ is 0.005 or better with a data acquisition time of $30 \mathrm{~s}$ at each $\theta$. Figure 3 shows examples of Muller matrices at three $\theta$ for the beetle C. aurata. A detailed description of Figure 3 can be found elsewhere. ${ }^{19}$ All calculations of derived parameters and all modeling were performed with CompleteEASE (J. A. Woollam Co., Inc.).

\subsection{Electromagnetic Modeling}

MMSE-data hold information about the structure of the beetle cuticle as well as about the optical functions of its constituents. Through electromagnetic modeling these properties can be quantified. The procedure is to design a multilayered model and assign each layer an optical dispersion function with or without optical anisotropy. The model is normally based on a priori knowledge about the structure, e.g. from analysis of microscopy images. A cuticle is composed of dielectric organic material which can be described with relatively simple optical functions and free carriers and electronic band gaps do not have to be considered. In the UV-part of the spectra one may expect some absorption bands from electronic transitions and in the infrared range there may be absorption bands due to vibration transitions. Bulk scattering may also result in losses and shows up as featureless absorption. However, in the spectral range used here it is sufficient to use a Cauchy function $n=A+B / \lambda^{2}+C / \lambda^{4}$ to describe the refractive indicies of the various layers in a cuticle. The parameters $A, B$ and $C$ are fit parameters and in most cases we set $C=0$ for cuticle refractive indicies. 


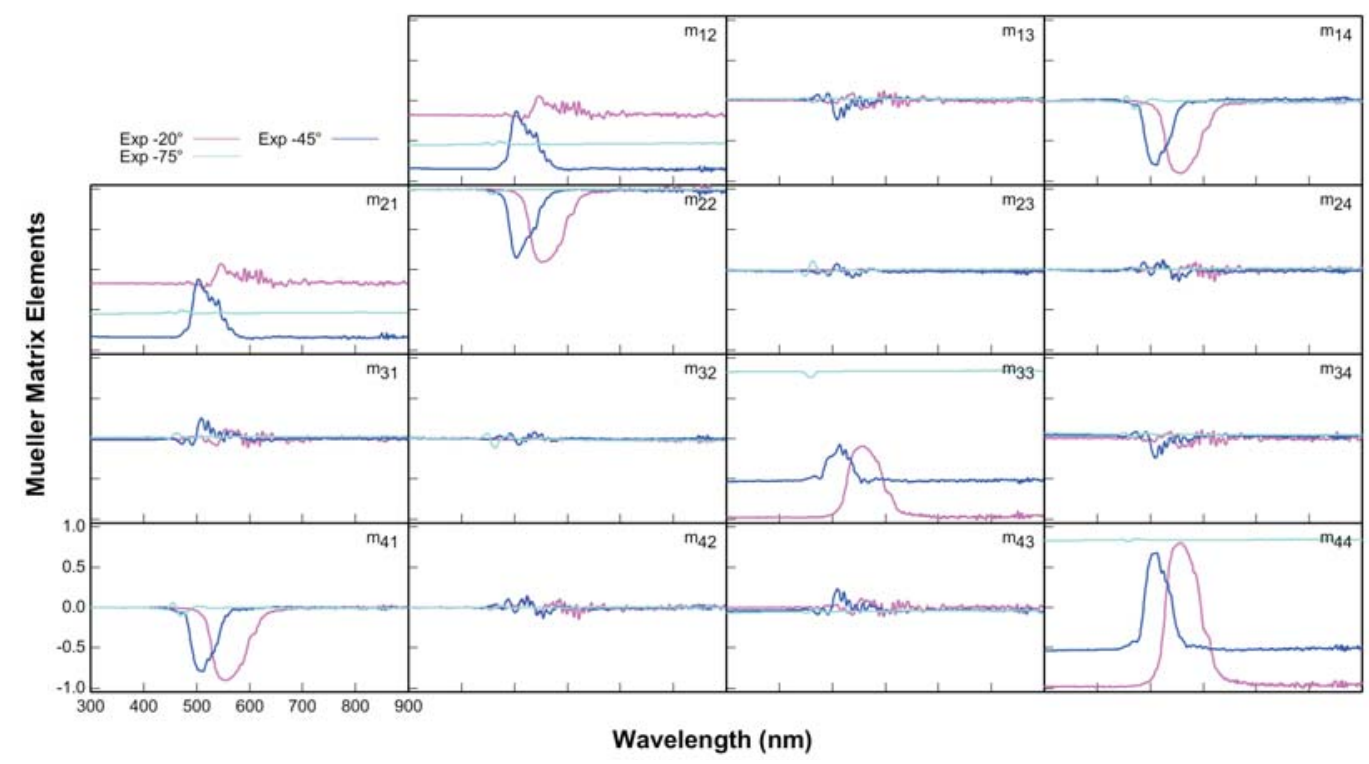

Figure 3. Example of Mueller-matrix spectra measured at $\theta=20^{\circ}, 45^{\circ}$ and $75^{\circ}$ on $C$. aurata.

When a structural model has been constructed, model fit parameters can be defined. The fit parameters are typically layer thicknesses and parameters in refractive index functions. These are assigned starting values and a forward calculation of a model Mueller matrix $\mathbf{M}^{\text {mod }}$ is performed and compared with the experimental Mueller matrix $\mathbf{M}^{\exp }$. After adjusting the fit parameters new forward calculations are performed until the difference between $\mathbf{M}^{\text {mod }}$ and $\mathbf{M}^{\text {exp }}$ is sufficiently small. After this manual fit procedure, a nonlinear regression procedure is used, e.g. a Levenberg-Marquardt algorithm, to minimize $\left|\mathbf{M}^{\bmod }-\mathbf{M}^{\exp }\right|$. When the global minimum is found, the current fit parameters are considered to be the result and provide a representative description of the sample. Further details about regression analysis in general are found elsewhere ${ }^{20}$ and specific details for analysis of a cuticle are given by Arwin et al. ${ }^{21}$

\subsection{Beetle Specimens}

In Table 1, the studied beetles are listed. All specimens were used without any treatment. Measurements were done on the scutellum as illustrated in Fig. 2 except for Cotinis mutabilis on which measurements were done on the abdominal side.

\begin{tabular}{lllll}
\hline Subfamily & Species & Auctor & Origin & Provider \\
\hline Cetoniinae & Cetonia aurata (greenish) & Linnaeus, 1758 & Sweden & Author J. L. \\
& Cetonia aurata (reddish) & Linnaeus, 1758 & Sweden & Author J. L. \\
& Coptomia laevis & Waterhouse, 1879 & Madagascar & MNH \\
& Cotinis mutabilis & Gory and Percheron, 1833 & Mexico & Author A. M.-G. \\
\multirow{5}{*}{ Rutelinae } & Anoplognathus aureus & Waterhouse, 1889 & Australia & MNH \\
& Chrysina argenteola & Bates, 1888 & Colombia & MNH \\
\hline Table 1. Overview of beetles studied in the family Scarabaeoidea. MNH=Museum of Natural History, Stockholm.
\end{tabular}

\section{RESULTS}

\subsection{Polarizing Properties}

\subsubsection{General introduction}

A beetle in its natural habitat is often exposed to scattered natural light incident from all directions. An observer will therefore at each viewing angle see a total irradiance which is the incident irradiance multiplied with the reflectance and summed over all incident angles. In direct sunlight the irradiance from the sun dominates 

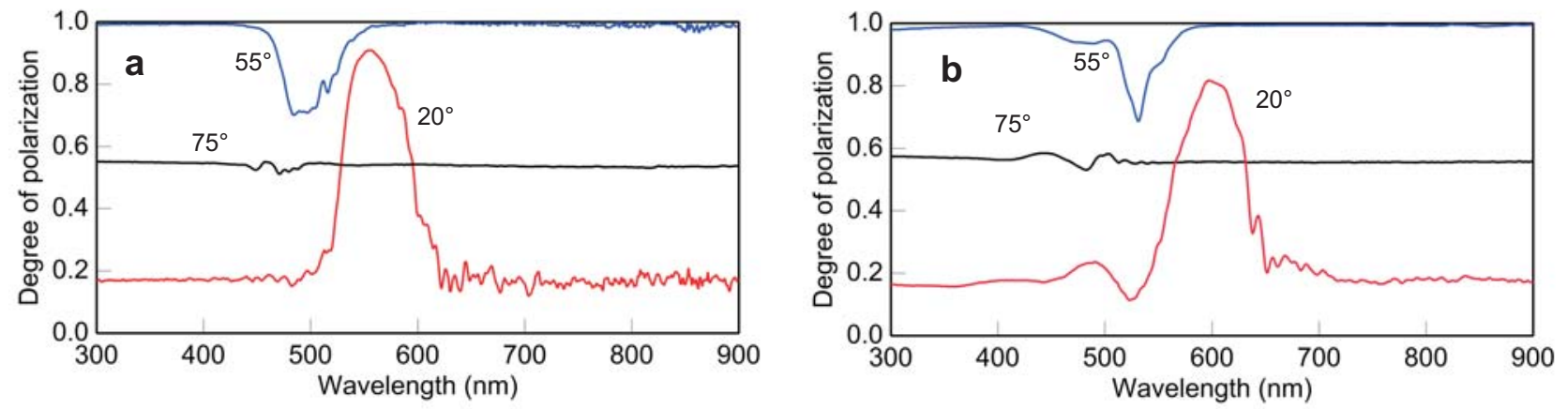

Figure 4. Degree of polarization for incident unpolarized light at three angles of incidence for a green (a) and a red (b) C. aurata.

and contributions from the background illumination may be neglected. Scattering effects may also contribute significantly. Some beetles like Cyphochilus insulanus (Moser, 1918) scatter light diffusely ${ }^{22}$ whereas other beetles including some of those studied here are more specularly reflecting. There are also beetles, e.g. C. gloriosa, with a structured cuticle which exhibit off-specular reflection in specific directions. ${ }^{23,24}$

The reflectance of a beetle cuticle depends largely on its structure. The cuticle is composed of chitin, proteins and other organic materials with relative low refractive indicies in the range $1.3-1.8 .^{21}$ If viewed as an ideal dielectric mirror, a cuticle would have a specular reflectance of the order of 0.04 at normal incidence and close to 1 at grazing incidence but surface and bulk scattering effects will lower these values. The cuticle of many beetles are multilayered reflectors and interference effects may strongly increase the reflectance. Simulations based on analytic expressions ${ }^{25}$ show, as an example, that a chiral multilayered structure (circular Bragg reflector) in a cuticle can have a reflectance close to $0.5 .^{26}$ For an observer who registers an irradiance we also have to consider source polarization and polarized vision of the observer.

\subsubsection{Degree of polarization}

In this report polarization features in reflected light from the cuticle of beetles are addressed. It is therefore important to check how large the degree of polarization of the reflected light is. In Fig. 4 we show $P$ calculated by using Eq. (7) for both a green and a red $C$. aurata as an example. As incident light we have chosen unpolarized light, i.e. worst case for exploring polarization capabilities of a cuticle. At near-normal incidence $\left(\theta=20^{\circ}\right)$, $P$ for the green specimen is below 0.2 except in the spectral region 500 and $620 \mathrm{~nm}$ where it is significantly larger with a peak value of 0.9 at $555 \mathrm{~nm}$. In this region the reflected light is near-circularly polarized as will be discussed in the next section. At $\theta=55^{\circ}$, the range 450-550 nm corresponds to the region with near-circular polarization (CP) which thus has shifted to shorter wavelengths. For $\lambda<450 \mathrm{~nm}$ and for $\lambda>550 \mathrm{~nm}$, the light is almost completely polarized $(P \approx 1)$ and the cuticle is here effectively a dielectric mirror. $\theta=55^{\circ}$ is equivalent to a Brewster angle for a dielectric material and the light is linearly polarized in the out-of-plane direction ( $s$-polarization). For larger $\theta$ the $\mathrm{CP}$ features decrease and at $\theta=75^{\circ}$, which is the largest $\theta$ we can use, they have almost vanished and $P \approx 0.55$ in the whole spectral range. For even larger $\theta$ we anticipate that $P$ monotonously decreases towards zero at grazing incidence. The red specimen has polarization features shifted towards longer wavelengths but is otherwise similar to the green specimen.

For the other beetles studied in this investigation, the $P$-characteristics are different compared to that in Fig. 4. This is illustrated by further examples in Fig. 5 which shows $P$ for $A$. aureus which is a broad-band chiral reflector and for $C$. laevis which has no or very small chiral structure. For $A$. aureus, $P$ is large at $\theta=20^{\circ}$ for $\lambda>500 \mathrm{~nm}$ and cuticle interference oscillations are very pronounced. $P$ for $C$. laevis only exhibit some small features around $550 \mathrm{~nm}$. Even though its cuticle most likely is multilayered, the data can be modeled as a bulk material with its refractive index given by a Cauchy dispersion relation $n=A+B / \lambda^{2}+C / \lambda^{4}$ with values as shown in Fig. 5b. 

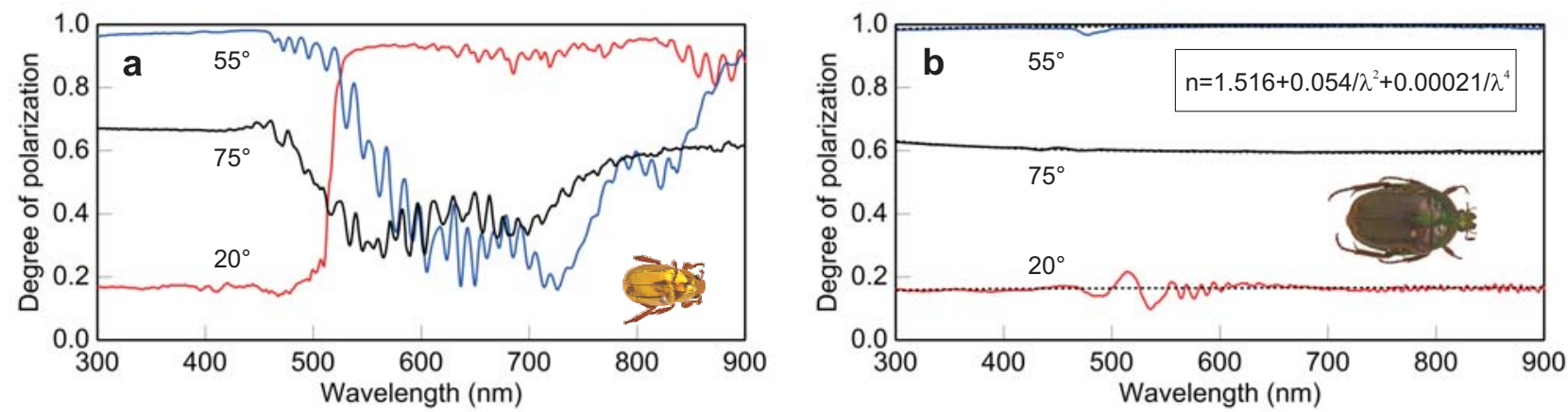

Figure 5. Degree of polarization for incident unpolarized light at three angles of incidence for A. aureus (a) and C. laevis (b). For $C$. laevis the MMSE data were fitted with a Cauchy dispersion model given by the insert and also shown by the dashed curves.
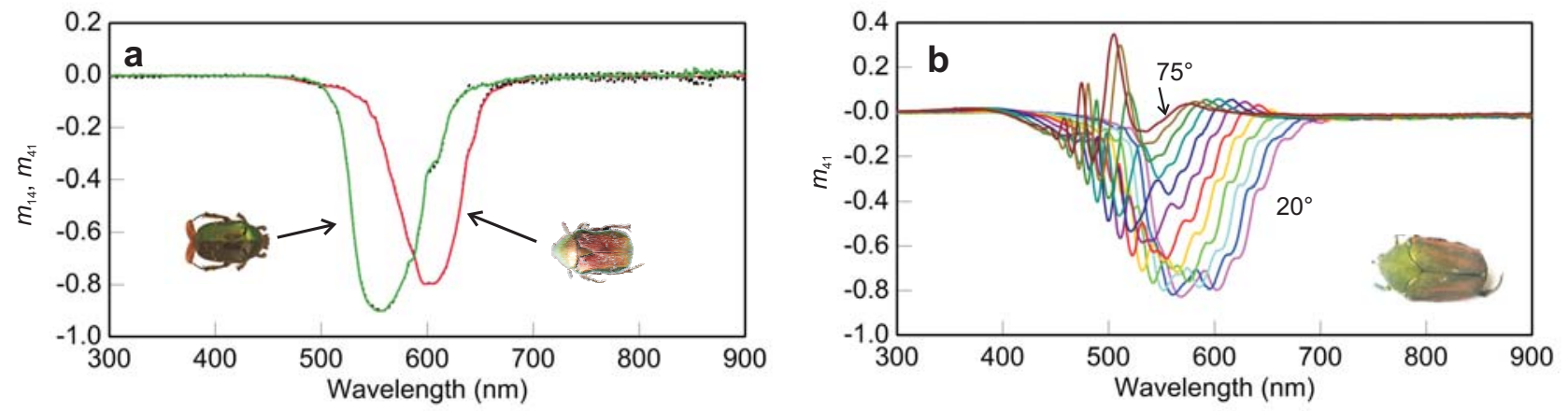

Figure 6. Mueller-matrix elements $m_{41}$ and $m_{14}$ at $\theta=20^{\circ}$ for a green and a red C. aurata (a). Mueller-matrix element $m_{41}$ for $C$. mutabilis at angles of incidence from $\theta=20^{\circ}$ to $75^{\circ}$ in steps of $5^{\circ}$ (b).

\subsubsection{Narrow-band chiral reflectors}

In Fig. 6a we show the Mueller-matrix elements $m_{41}$ and $m_{14}$ for a green and a red C. aurata at $\theta=20^{\circ}$. It is seen that $m_{41}$ is very close to $m_{14}$ indicating a symmetric Mueller matrix in accordance with symmetries found in Mueller matrices of chiral systems. ${ }^{19,27}$ In Fig. 6 we also show $m_{41}$ for C. mutabilis at several angles of incidence and a shift of features towards shorter wavelengths with $\theta$ is seen as well as a decrease in the magnitude of $m_{41}$. The element $m_{41}$ is an indicator of circular polarization capability and corresponds to the degree of circular polarization $P_{c}$ (as defined in Eq. (8)) in reflected light when the cuticle is illuminated with unpolarized light. The negative sign of $m_{41}$ shows that the polarization is left-handed for $C$. aurata. How close to circular polarization the reflected light is depends also on the linear polarization contributions in $m_{21}$ and $m_{31}$ and is described with the ellipticity angle $\epsilon$ in Eq. (9). The ellipticity angle $\epsilon$ is presented in Fig. 7 for the same three beetles as in Fig. 6 and it is seen that $\epsilon$ is close to $-45^{\circ}$ but in slightly different spectral ranges for the three beetles.

\subsubsection{Broad-band chiral reflectors}

In Fig. 8a we show the polarizance vector $\mathbf{P}$ for $C$. argenteola. The element $m_{41}$ differs from those for the $C$. aurata beetles in Fig. 6 as it is non-zero over the whole visible spectrum and not only in a band of the spectrum corresponding to green or red color. Weak interference oscillations are seen in $m_{41}$ for $\lambda>600 \mathrm{~nm}$ but are more pronounced in $m_{21}$ and $m_{31}$ for which they are seen for $\lambda>400 \mathrm{~nm}$. The ellipticity angle $\epsilon$ calculated with Eq. (9) from $\mathbf{P}$ at $\theta=20^{\circ}$ is shown in Fig. 8b and we observe that $\epsilon \approx-40^{\circ}$ in the whole visible spectrum but with some weak oscillations. A value of $\epsilon=-40^{\circ}$ is considered to correspond to near-circular polarization and its negative sign at $\theta=20^{\circ}$ shows that the light is left-handed polarized. 


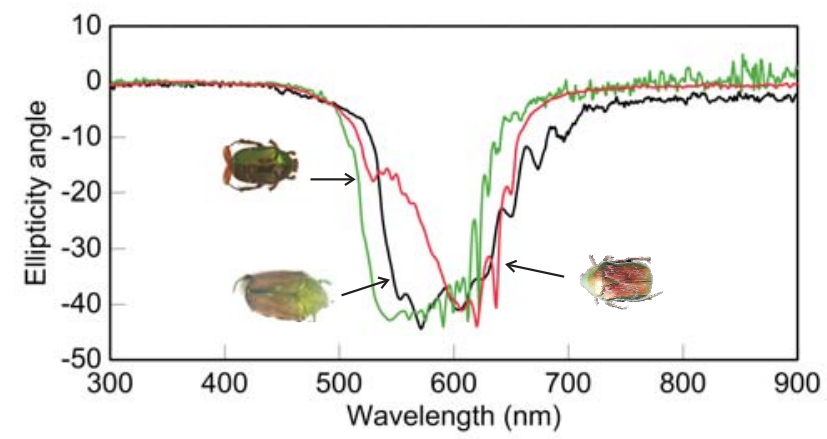

Figure 7. Ellipticity angle at $\theta=20^{\circ}$ for a green and a red C. aurata and for C. mutabilis.
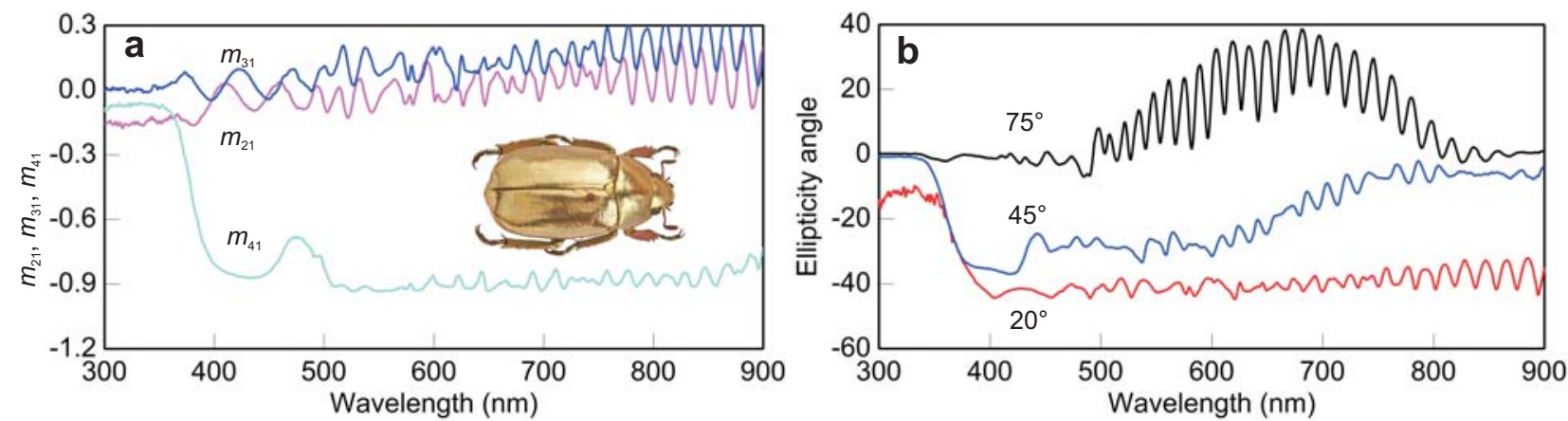

Figure 8. The polarizance vector $\mathbf{P}=\left[m_{21}, m_{31}, m_{41}\right]^{T}$ at $\theta=20^{\circ}$ (a) and ellipticity angle at $\theta=20^{\circ}, 45^{\circ}$ and $75^{\circ}(\mathrm{b})$ for C. argenteola.

\subsubsection{Left-handed and right-handed polarized reflection}

In Fig. $8 \mathrm{~b}$ we show the ellipticity angles for $C$. argenteola not only at $\theta=20^{\circ}$ but also at $\theta=45^{\circ}$ and $75^{\circ}$. Interestingly we can observe that $\epsilon$ is positive for $\theta=75^{\circ}$ with a maximum of around $40^{\circ}$ at $\lambda=650 \mathrm{~nm}$, i.e. the reflected light has right-handed and near-circular polarization, whereas at $\theta=20^{\circ} \epsilon$ is around $-40^{\circ}$, i.e. the polarization is left-handed and near circular. For intermediate angles (only $\theta=45^{\circ}$ is shown) $\epsilon$ becomes less negative compared to at $\theta=20^{\circ}$ and when the angle of incidence varies from near-normal to grazing incidence, $\epsilon$ varies from negative to positive values in the spectral range $500-800 \mathrm{~nm}$. We conclude that $C$. argenteola when illuminated with natural light will reflect left-handed polarized light in the whole visible spectrum at near-normal incidence and right-handed polarized light for $\lambda>500 \mathrm{~nm}$ at large angles of incidence.

\subsection{Modeling}

\subsubsection{Regression analysis for structural characterization}

Figure 9 shows the structural model we use to model the color-generating part of the cuticle of $C$. aurata. The top epicuticle is modeled as a uniaxial layer with thickness $d_{e p i}$ and with the extraordinary axis perpendicular to the surface. The ordinary as well as the extraordinary refractive indicies are described with Cauchy dispersion functions. The exocuticle is modeled assuming a fixed thickness of $d_{e x o}=8 \mu \mathrm{m}$ divided into 360 biaxial sublayers. All sublayers have equal thickness but each consecutive layer is rotated an angle $\Delta \phi$ with respect to the previous layer. The number of turns $T$ is assigned as fit parameter and the pitch $\Lambda$ indicated in Fig. 9 is obtained as $\Lambda=d_{\text {exo }} / T$. To account for inhomogeneities a distribution $\Delta T$ of the number of turns is included. All sublayers have the same biaxial refractive index and are modeled with Cauchy dispersion functions similar to the epicuticle. A small absorption is also included in refractive indices of both the epicuticle and exocuticle. The absorption of the epicuticle and the exocuticle implies that the underlying endocuticle has no influence on the modeling. Taken all together we use 13 fit parameters including $d_{\text {epi }}, T, \Delta T$ and the parameters of the refractive indices of the epicuticle and exocuticle as detailed elsewhere. ${ }^{21}$ 

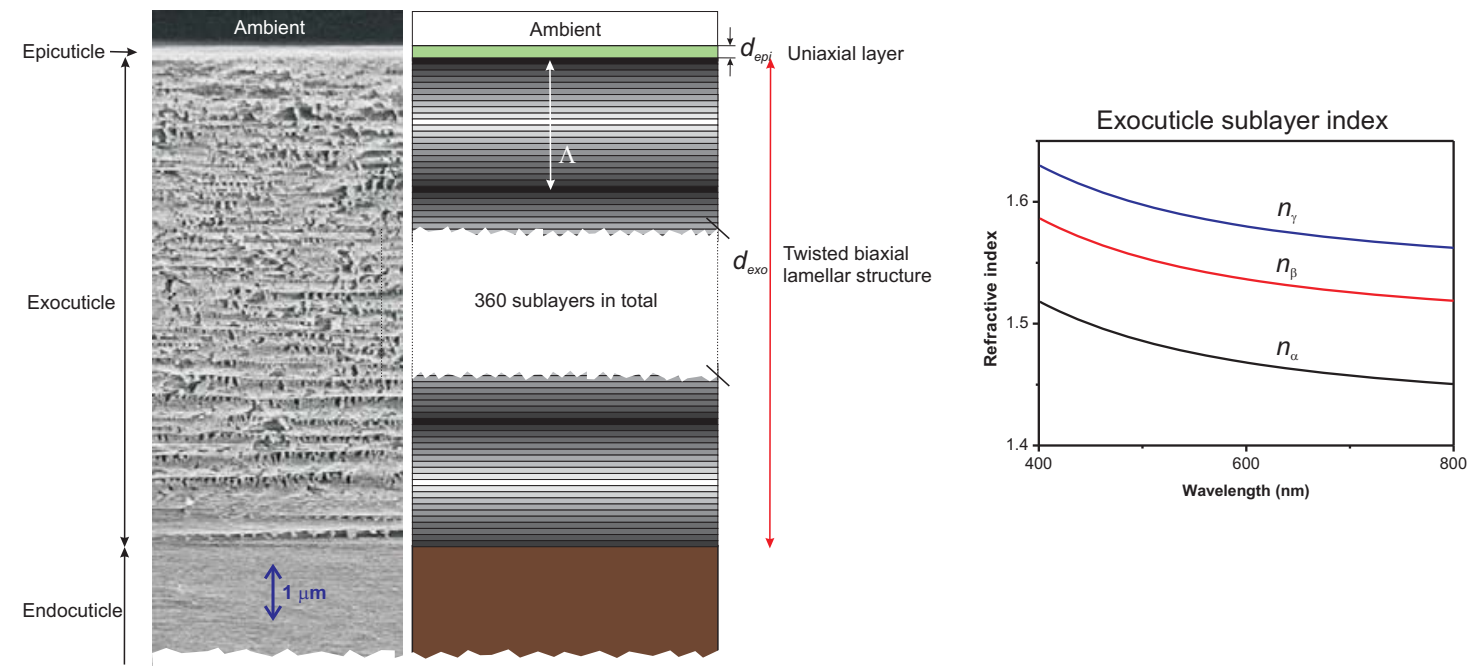

Figure 9. A schematic view of the structural model used for analyzing MMSE-data. Black and white sublayers in the exocuticle indicate biaxial layers for which the corresponding optic axes are perpendicular whereas the grayscale illustrates intermediate orientations. To the left a scanning electron microscopy image illustrates the multilayered structure of the cuticle of a $C$. aurata specimen. To the right the modeled biaxial refractive index of the sublayers in the exocuticle is shown.

In Fig. 10 we show an example of best fit between MMSE experimental data $\mathbf{M}^{\exp }$ and model-generated data $\mathbf{M}^{\text {mod }}$ for measurements on $C$. aurata. There are not two identical beetles and for the specimen used to record the data in Fig. 10 we find $d_{e p i}=544 \mathrm{~nm}, T=21.1$ which gives a pitch $\Lambda=379 \mathrm{~nm}$. The best fit biaxial refractive index of the exocuticle is shown as $n_{\alpha}, n_{\beta}$ and $n_{\gamma}$ in Fig. 9 and it is seen that it decreases with wavelength and that $n_{\gamma}$, the index perpendicular to the cuticle surface, is largest. The value on the pitch was found to vary from $370 \mathrm{~nm}$ for a blue specimen to $387 \mathrm{~nm}$ for a red specimen of C. aurata. $^{28}$

\subsubsection{Phenomenological modeling of cuticle structures}

Besides regression analysis for structural characterization of the cuticle of beetles, another approach considers the optical modes propagating in cuticle structures at oblique incidence. ${ }^{29}$ Particularly, we have analyzed the spectral dependence of maxima and minima in the interference oscillations observed in MMSE data of $C$. mutabilis. As an example, Fig. 11 shows the polarizance vector measured at $\theta=25^{\circ}$ where four spectral regions (I-IV) are identified from the change in the frequency of interference oscillations in $m_{21}$ and $m_{31}$ as well as from the spectral characteristics of the band of selective reflection of left-handed polarized light $\left(m_{41}<0\right)$. From the analysis, a structural model is proposed which is comprised of slabs organized in two chiral stacks of thicknesses $d_{1}$ and $d_{2}$ which together corresponds to the outer part of the exocuticle. There is also an inner exocuticle and an epicuticle. ${ }^{29}$ Thus, the spectral regions I and IV correspond to optical modes propagating without attenuation and from data in region I, an estimate of the outer exocuticle thickness $d_{1}+d_{2}$ can be obtained which for the cuticle studied in Fig. 11 is $10.3 \mu \mathrm{m}$. Left-handed polarized light is selectively reflected in spectral regions II and III from the chiral stacks located respectively, at the top and bottom of outer exocuticle.

\section{DISCUSSION}

Only specular reflection from beetles has been addressed in this report. As mentioned in the introduction some beetles in the Cyphochilus family can scatter diffusely and have a white appearance ${ }^{22}$ and the degree of polarization is low for normal incidence of unpolarized light. However, for large angles of incidence also these beetles act as polarizers. ${ }^{30}$ Another type of reflection is found in C. gloriosa. When illuminated with incident light at $\theta=45^{\circ}$, this beetle reflects light with near-circular left-handed polarization in a direction $30^{\circ}$ to $40^{\circ}$, i.e. closer to the normal compared to incident light. This occurs on the green areas of the cuticle of this striped beetle. The other stripes are gold-colored and reflect similar to C. argenteola. ${ }^{23}$ 


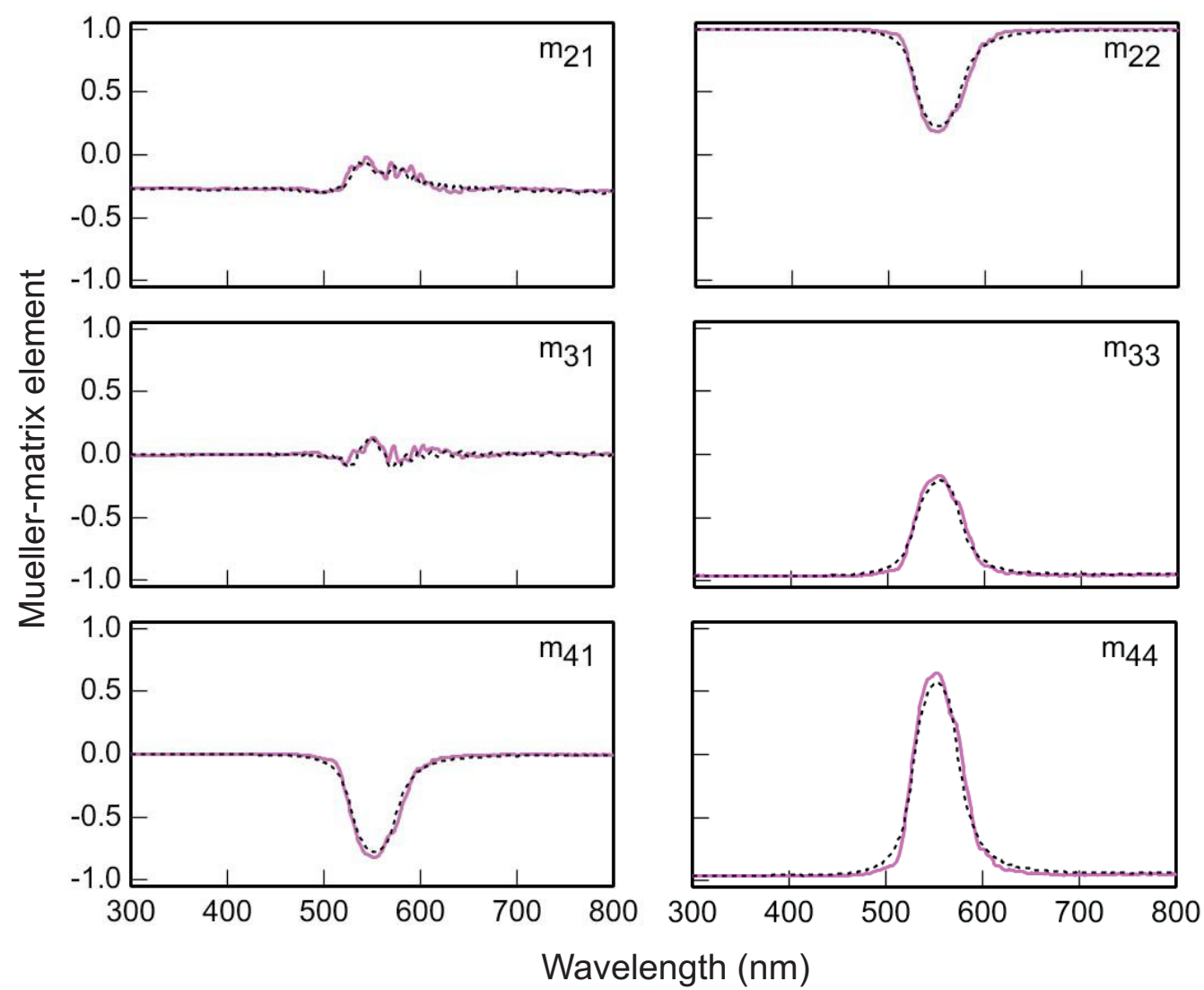

Figure 10. Selected measured Mueller-matrix elements (solid curves) and model-generated elements (dashed curves) for a green $C$. aurata at $\theta=25^{\circ}$. Only elements of the polarizance vector $\mathbf{P}=\left[m_{21}, m_{31}, m_{41}\right]^{T}$ and the diagonal elements $m_{22}, m_{33}$ and $m_{44}$ of the full matrix are shown. The elements of the diattenuation vector $\mathbf{D}=\left[m_{12}, m_{13}, m_{14}\right]^{T}$ are almost identical to those of $\mathbf{P}$ and the remaining elements are close to zero. All graphs have the same scales as shown to the left and at the bottom.

As shown in this report, a cuticle can polarize incident unpolarized light and the polarization of the reflected light will depend on incident angle and wavelength. Conversely if a cuticle is illuminated with polarized light depolarizing may occur which also depends on incident angle and wavelength. A measured Mueller matrix contains information about this depolarization. Depolarizing Mueller matrices can be sum decomposed which provides a phenomenological insight into the character of the reflector. Pioneering work was done by Ossikovski et al. $^{31}$ who showed that sum decomposition of single wavelength Mueller-matrix images measured on beetles could be done. They found that the cuticle studied can be characterized as a nondiagonal depolarizer, a type of depolarizer which is not very common. We have extended this type of analysis to spectral Mueller matrices and have shown that a Mueller matrix measured at near-normal incidence on $C$. aurata can be described as a weighted sum of Mueller matrices of an ideal left-handed circular polarizer and an ideal dielectric mirror. ${ }^{26}$

The Mueller-matrix data presented in this report were recorded with a spot size of $100 \mu \mathrm{m}$ or smaller. However, many beetles have a cuticle which is strongly curved and rich in irregularities. Some beetles are patterned with pattern features in the order of $10 \mu \mathrm{m}$ which are much smaller than the stripes in the cuticle of C. gloriosa. A smaller spot size would therefore be a welcomed methodology development which would allow to explore additional details in polarizing properties of cuticle reflection. Single-wavelength imaging ${ }^{26,31}$ of a cuticle at near-normal incidence to record Muller matrices can be performed with higher spatial resolution compared to that obtained by using a focused beam. Hopefully future development will lead to instruments providing not only single-wavelength images but also allow spectral imaging in a wide range of angles of incidence to facilitate a comprehensive mapping of polarization features. 

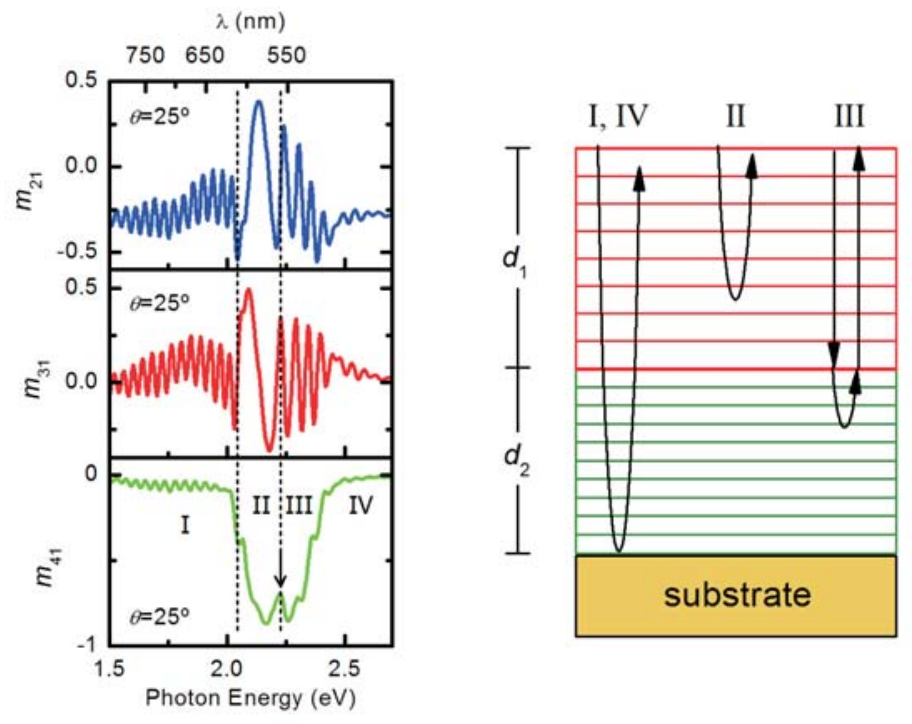

Figure 11. Measured polarizance vector $\mathbf{P}=\left[m_{21}, m_{31}, m_{41}\right]^{T}$ for C. mutabilis at an angle of incidence of $25^{\circ}$ (left). In the spectral regions indicated by I, II, III and IV, different optical modes propagate as indicated in the proposed structural model (right).

\section{CONCLUSIONS}

Mueller-matrix ellipsometry can provide a full description of the polarization features of specular light reflection from the cuticle of beetles. Specifically we have shown that:

- a high degree of polarization in reflected light can be found in the color bands of the cuticle of beetles for incident natural light;

- C. aurata and C. mutabilis exhibit narrow-band near-circular polarization features;

- A. aureus and C. argenteola exhibit broad-band polarization features;

- the polarization handedness depends on angle of incidence;

- extraction of structural parameters of the cuticle of beetles by electromagnetic modeling of Mueller-matrix data is feasible; and

- description of cuticle structure from cuticle interference patterns is possible.

\section{ACKNOWLEDGMENTS}

Specimens of $C$. argenteola, C. aureus and C. laevis were on loan from Museum of Natural History in Stockholm. Jens Birch is acknowledged for providing photos of the beetles and Torun Berlind for the SEM image. This work is supported by grants from the Swedish Research Council, Carl Tryggers foundation and the Swedish Government Strategic Research Area in Materials Science on Functional Materials at Linköping University (Faculty Grant SFO-Mat-LiU \# 2009-00971). Knut and Alice Wallenberg foundation is acknowledged for support to instrumentation. 


\section{REFERENCES}

1. A. E. Seago, P. Brady, J.-P. Vigneron, and T. D. Schultz, "Gold bugs and beyond: a review of iridescence and structural colour mechanisms in beetles (Coleoptera)," J. R. Soc. Interface 6, pp. S165-S184, 2009.

2. G. P. Horváth and D. Varjú, Polarized Light in Animal Vision: Polarization Patterns in Nature, Springer, Heidelberg, second ed., 2004.

3. M. Dacke, P. Nordström, and C. H. Scholtz, "Twilight orientation to polarised light in the crepuscular dung beetle Scarabaeus zambesianus," J. Exp. Biol. 206, pp. 1535-1543, 2003.

4. P. Brady and M. Cummings, "Differential response to circularly polarized light by the Jewel scarab beetle Chrysina gloriosa," Am. Nat. 175, pp. 614-620, 2010.

5. M. Blahó, A. Egri, R. Hegedüs, J. Jósvai, M. Tóth, K. Kertész, L. P. Biró, G. Kriska, and G. Horváth, "No evidence for behavioral responses to circularly polarized light in four scarab beetle species with circularly polarizing exocuticle," Physiol. Behav. 105, pp. 1067-1075, 2012.

6. A. Sweeney, C. Jiggins, and S. Johnsen, "Polarized light as a butterfly mating signal," Nature 423, pp. 31-32, 2003.

7. A. A. Michelson, "On metallic colouring in birds and insects," Phil. Mag. 21, pp. 554-567, 1911.

8. S. Caveney, "Cuticle reflectivity and optical activity in scarab beetles: the role of uric acid," Proc. R. Soc. London, Ser. B 178, pp. 205-225, 1971.

9. D. H. Goldstein, "Polarization properties of Scarabaeidae," Appl. Opt. 45, pp. 7944-7950, 2006.

10. H. Arwin, R. Magnusson, J. Landin, and K. Järrendahl, "Chirality-induced polarization effects in the cuticle of scarab beetles: 100 years after Michelson," Phil. Mag. 12, pp. 1583-1599, 2012.

11. I. Hodgkinson, S. Lowrey, L.Bourke, A. Parker, and M. W. McCall, "Mueller-matrix characterization of beetle cuticle: polarized and unpolarized reflections from representative architectures," Appl. Opt. 49, pp. 45584567, 2010.

12. M. Srinivasarao, "Nano-optics in the biological world: Beetles, butterflies, birds, and moths," Chem. Rev. 99, pp. 1935-1961, 1999.

13. M. Sarrazin, P. Lambin, and S. Berthier, "Living light: Uniting biology and photonics - a memorial meeting in honour of prof Jean-Pol Vigneron," Mat. Today: Proceedings 1, pp. 107-228, 2014.

14. G. P. Horváth, Polarized Light and Polarization Vision in Animal Sciences, Springer, Heidelberg, second ed., 2014.

15. T. Lenau and M. Barfoed, "Colours and metallic sheen in beetle shells - a biomimetic search for material structuring principles causing light interference," Adv. Eng. Mat. 10, pp. 299-314, 2008.

16. A. R. Parker and H. E. Townley, "Biomimetics of photonic nanostructures," Nature Nanotechn. 2, pp. 347$353,2007$.

17. R. Magnusson, J. Birch, C.-L. Hsiao, P. Sandström, H. Arwin, and K. Järrendahl, " $\mathrm{In}_{x} \mathrm{Al}_{1-x} \mathrm{~N}$ chiral nanorods mimicking the polarization features of scarab beetles," This Proceedings -, 2015.

18. D. H. Goldstein, Polarized Light, CRC Press, Boca Raton, third ed., 2010.

19. H. Arwin, R. Magnusson, L. Fernández del Río, C. Åkerlind, E. Muñoz-Pineda, J. Landin, A. MendozaGalván, and K. Järrendahl, "Exploring optics of beetle cuticles with Mueller-matrix ellipsometry," Mater. Today Proc. 1S, pp. 155-160, 2014.

20. H. Fujiwara, Spectroscopic ellipsometry: principles and applications, John Wiley \& Sons Ltd., Chichester, 2007.

21. H. Arwin, T. Berlind, B. Johs, and K. Järrendahl, "Cuticle structure of the scarab beetle Cetonia aurata analyzed by regression analysis of Mueller-matrix ellipsometric data," Opt. Express 21, pp. 22645-22656, 2013.

22. P. Vukusic, B. Hallam, and J. Noyes, "Brilliant whiteness in ultrathin beetle scales," Science 315, p. 348, 2007.

23. L. Fernández del Río, H. Arwin, and K. Järrendahl, "Polarizing properties and structural characteristics of the cuticle of the scarab beetle Chrysina gloriosa," Thin Solid Films 571, pp. 410-415, 2014.

24. V. Sharma, M. Crne, J. O. Park, and M. Srinivasarao, "Structural origin of circularly polarized iridescence in Jeweled beetles," Science 449-451, p. 325, 2009. 
25. A. Lakhtakia and M. McCall, "Simple expressions for Bragg reflection from axially excited chiral sculptured thin films," J. Modern Opt. 49, pp. 1525-1535, 2002.

26. H. Arwin, R. Magnusson, E. Garcia-Caurel, C. Fallet, K. Järrendahl, M. Foldyna, A. D. Martino, and R. Ossikovski, "Sum decomposition of Mueller-matrix images and spectra of beetle cuticles," Opt. Express 23, pp. 1951-1966, 2015.

27. E. Muñoz-Pineda, K. Järrendahl, H. Arwin, and A. Mendoza-Galván, "Symmetries and relationships between elements of the Mueller matrix spectra of the cuticle of the beetle Cotinis mutabilis," Thin Solid Films 571, pp. 660-665, 2014.

28. H. Arwin, L. Fernández del Río, and K. Järrendahl, "Comparison and analysis of Mueller-matrix spectra from exoskeletons of blue, green and red Cetonia aurata," Thin Solid Films 571, pp. 739-743, 2014.

29. A. Mendoza-Galván, E. Muñoz-Pineda, K. Järrendahl, and H. Arwin, "Evidence for a dispersion relation of optical modes in the cuticle of the scarab beetle Cotinis mutabilis," Opt. Mat. Express 4, pp. 2484-2496, 2014.

30. C. Åkerlind, H. Arwin, T. Hallberg, J. Landin, J. Gustafson, and K. Järrendahl, "Scattering and polarization properties of the scarab beetle Cyphochilus insulanus," Manuscript -, pp. -, 2015.

31. R. Ossikovski, M. Foldyna, C. Fallet, and A. Martino, "Experimental evidence for naturally occurring nondiagonal depolarizers," Opt. Lett. 34, pp. 2426-2428, 2009. 